M. G. Marinari

Nagoya Math. J.

Vol. 70 (1978), 105-110

\title{
EXAMPLES OF BAD NOETHERIAN LOCAL RINGS
}

\author{
MARIA GRAZIA MARINARI
}

In this paper we use a general technique, due to Larfeldt-Lech [9], to show that there exist local noetherian rings which are "bad" with respect to some properties which are obviously verified in the algebrogeometric case. In particular we show that there exist local Gorenstein rings which are not homomorphic images of regular (local) rings and that there exist local rings which do have canonical module but do not have canonical algebra.

We wish to thank Dr. D. Eisenbud for his smart suggestions and kind help in the conception and preparation of this paper.

\section{Introduction}

We recall some definitions and well-known facts about noetherian local rings (all rings are assumed to be commutative with 1).

Let $R$ be a noetherian local ring with maximal ideal $\mathfrak{m}$. Its completion $\hat{R}$ can be written as $\hat{R}=S / \mathfrak{a}$ where $S$ is a regular local ring and $\mathfrak{a} \subset S$ is an ideal (see [2] $\S 6$ ). The invariant $d(R)$ is defined as follows: $d(R)=\mu(\mathfrak{a})-(\operatorname{dim} S-\operatorname{dim} R)$ where $\mu(\mathfrak{a})$ is the minimal number of generators for $\mathfrak{a}$; $d(R)$ does not depend on the chosen representation $\hat{R}=S / \mathfrak{a}$. Incidentally we have the following facts: $R$ is a complete intersection iff $d(R)=0, R$ is an almost complete intersection iff $d(R)=1$, moreover $d(R)=1$ implies $R$ is not Gorenstein (see [8] $\S 1$ ). Let $(R, \mathfrak{m}, k)$ be a noetherian local ring. A finitely generated $R$-module $G$ is said to be a Gorenstein $R$-module if there exists a non-negative integer $n$ such that $\operatorname{Ext}_{R}^{i}(k, G)=0$ for $i \neq n$. If moreover $n=\mathrm{ht}_{G} \mathfrak{m}$ and $\mu^{i}(\mathfrak{m}, G)=\operatorname{dim}_{k} \operatorname{Ext}_{R}^{i}(k, G)=\delta_{i, n}$ (here necessarily $n=\mathrm{ht}_{R} \mathfrak{m}$, i.e. $R$ is a Cohen-Macaulay ring (see [12] $\S 3$ )), then $G$ is said to be a Gorenstein module of rank 1 (for more details on rank see [3] §3), or even canonical module of $R$, or dualizing module of $R$. In [7] $\S 5$ it is shown that if

Received October 19, 1976.

Revised April 2, 1977. 
$R$ has canonical module then this is uniquely determined up to isomorphism, and therefore it is denoted by $K_{R}$. Using the above notation we have $K_{R} \otimes_{R} \hat{R} \simeq K_{\hat{R}} \simeq \operatorname{Ext}_{S}^{t}(\hat{R}, S)$ where $t=\operatorname{dim} S-\operatorname{dim} R$. Moreover, if $R$ is a (local) Cohen-Macaulay ring (from now shortly C.M.) then there exists $K_{R}$ iff $R$ can be expressed as a homomorphic image of a (local) Gorenstein ring (see [10] and [12]). Let again $R$ and $\hat{R}$ be as above. Let $\left\{a_{1}, \cdots, a_{m}\right\}$ be a minimal system of generators for $a$ in $S$ and let $E=S\left\langle Z_{1}, \cdots, Z_{m} ; d Z_{i}=a_{i}\right\rangle$ be the Koszul complex of $S$ with respect to $\left\{a_{1}, \cdots, a_{m}\right\}$. An $R$-algebra which is finitely generated as an $R$-module and whose completion is isomorphic to the homology algebra $H(E)$ of $E$ is called canonical algebra of $R$ (see [6] §2). In [6] loc. cit. it is shown that if $R$ has canonical algebra then this last is uniquely determined up to isomorphism, therefore it is denoted by $A(R)$. If $R$ can be expressed as a homomorphic image of a regular (local) ring $S$, and $d(R)=\delta$, then there exists $A(R)$ and also $A_{\delta}(R)=K_{R}, A_{i}(R)=0$ for $i>\delta$. Moreover canonical algebras distinguish complete intersections from Gorenstein rings (in fact $R$ is a Gorenstein ring iff $K_{R}=R$ and it is a complete intersection iff $A(R)=R$ (see [7] and [8])).

We conclude this part carrying explicitly the proof of the following known fact since we can not give an exact reference for it.

Proposition 1.1. Let $R$ be a noetherian local integral domain which is a homomorphic image of a regular (local) ring. Then $\hat{R}$ is generically a complete intersection (i.e. for every minimal prime $\mathfrak{q} \subset \hat{R}, \hat{R}_{\mathfrak{q}}$ is a complete intersection).

Proof. Let $R=S / \mathfrak{p}, S$ regular (local) ring, $\mathfrak{p} \subset S$ prime ideal. $S_{\mathfrak{p}}$ is regular, hence $\mathfrak{p} S_{\mathfrak{p}}=\left(a_{1}, \cdots, a_{r}\right) S_{\mathfrak{p}}$, with $r=\mathrm{ht} \mathfrak{p}, a_{i} \in \mathfrak{p}$. Then, if $\mathfrak{p}=\left(u_{1}, \cdots, u_{n}\right) S$ with $n>r$, there exists some $f \in S-\mathfrak{p}$ such that $f u_{i}$ $\in\left(a_{1}, \cdots, a_{r}\right) S$ for every $i$, hence $\mathfrak{p} S_{f}=\left(a_{1}, \cdots, a_{r}\right) S_{f}$. This means $R_{f}$ is a complete intersection. Therefore $\hat{R}_{\tilde{f}}$ is a complete intersection. Moreover, since for every minimal prime $\mathfrak{q} \subset \hat{R}$ we have $\hat{R}_{\mathfrak{q}}=\left(\hat{R}_{\mathcal{f}}\right)_{\mathfrak{q} \hat{R}_{f}}$, our contention is completely proved.

\section{The Larfeldt-Lech construction and examples}

In this paragraph we give some examples of bad noetherian local rings, more precisely we will construct:

a) a class of local 1-dimensional C. M. rings which do not have canonical module 
b) a class of local 1-dimensional Gorenstein rings which can not be expressed as homomorphic images of regular (local) rings

c) a class of local 1-dimensional Gorenstein rings which do not have canonical algebra.

All these examples are founded upon a particular case of the construction given in [9] theorem 1 . So we think it useful to sketch here briefly the aforesaid construction with regard to the special situation we are interested in. This especially because we do not know whether the Larfeldt-Lech paper has been already published and also because their construction, in the restrictive hypotheses we need, looks much clearer than in the general case.

Precisely we will deal with the following situation:

Let $k=\bar{k}$ be a field and let $Z$ be a finite set of indeterminates. Let $Q_{0}=k$ and $Q_{1}=k[[Z]] / \mathfrak{a}$ be a local artinian $k$-algebra. Clearly $\left(Q_{0}, Q_{1}\right)$ is a flat couple of 0 -dimensional local rings. Let $Q_{2}=\hat{Q}_{1}[[X]]$ $=Q_{1}[[X]]$ be the formal power series ring over $Q_{1}$ in one indeterminate. It is possible to show that there exists a 1-dimensional (noetherian) local domain $Q$ whose completion $\hat{Q} \simeq Q_{2}$.

For this, let $\mathfrak{m}_{i}=\max Q_{i}, i=0,1,2 ; K_{i}=Q_{i} / \mathfrak{m}_{i} \simeq k$. Let $u=\left\{u_{i}: i \in I\right\}$ be a set of generators for the ring extension $Q_{1}$ over $Q_{0} ; \# I \leq \aleph_{0}$ and tr. deg. $k[[X]] / k[X] \geqslant \boldsymbol{\aleph}_{0}$ so we can choose $\alpha: I \rightarrow k[[X]]$ so that we have an injection of $I$ into a transcendence basis of $k[[X]]$ over $k[X]$. Put $u^{*}=\left\{u_{i}+\alpha(i): i \in I\right\} \subset Q_{1}[[X]]$. Form the subring $R=k\left[u^{*}, X, X^{-1}\right]$ of $Q_{2}\left[X^{-1}\right]$. Put $T=R \cap Q_{2}$ and $Q=T_{T \cap \mathrm{m}_{2}}$. We want to show that $Q_{2}$ can be identified with the completion of $Q$ and that $\mathfrak{m}_{1} Q_{2} \cap Q=(0)$.

We will see that for this it suffices to prove

1. $\left(Q \cap \mathfrak{m}_{2}\right) Q_{2}=\mathfrak{m}_{2}$,

2. $Q \cap \mathfrak{m}_{2}^{n}=\left(Q \cap \mathfrak{m}_{2}\right)^{n}, n=1,2, \cdots$,

3. $Q \cap \mathfrak{m}_{1} Q_{2}\left[X^{-1}\right]=(0)$.

Clearly 1.-3. can be deduced from the following:

$1^{\prime} . \quad\left(T \cap \mathfrak{m}_{2}\right) Q_{2}=\mathfrak{m}_{2}$,

$2^{\prime} . \quad T \cap \mathfrak{m}_{2}^{n}=\left(T \cap \mathfrak{m}_{2}\right)^{n}, n=1,2, \cdots$,

$3^{\prime} . \quad T \cap \mathfrak{m}_{1} Q_{2}\left[X^{-1}\right]=(0)$.

$1^{\prime}$. Let $f \in T=k\left[u^{*}, X, X^{-1}\right] \cap Q_{2}$. Then $f$ can be written as $f=a_{0}$ $+a_{1} X+a_{2} X^{2}+\cdots f \in Q_{1}[[X]]$ and $f \in \mathfrak{m}_{2}$ iff $a_{0} \in \mathfrak{m}_{1}$. We can find finitely many elements $f_{1}, \cdots, f_{r} \in T$ such that their constant terms generate $\mathfrak{m}_{1}$ in $Q_{1}$. Then for an arbitrary $f \in T \cap \mathfrak{m}_{2}$ there exist $g_{1}, \cdots, g_{r} \in T$ such 
that $f-\sum_{i=1}^{r} f_{i} g_{i}$ has constant term 0 , i.e. it is divisible by $X$ in $T$, and this means $T \cap \mathfrak{m}_{2}=\left(f_{1}, \cdots, f_{r}, X\right)$. From this fact it follows that $\left(T \cap \mathfrak{m}_{2}\right) Q_{2}=\mathfrak{m}_{2}$, which is what we need.

$2^{\prime}$. Let $f \in T$. Then we have $f \in \mathfrak{m}_{2}^{n}$ iff its coefficients satisfy the conditions $a_{i} \in \mathfrak{m}_{1}^{n-i}$ (i.e. $\left.a_{i} X^{i} \in \mathfrak{m}_{2}^{n}\right)$ for $0 \leqslant i \leqslant n$, and this implies $f \in(T$ $\left.\cap \mathfrak{m}_{2}\right)^{n}$.

$3^{\prime}$. It is clear that because of $u^{*}$ no non-zero $f \in T$ can have all of its coefficients in $\mathfrak{m}_{1}$, so $T \cap \mathfrak{m}_{1} Q_{2}\left[X^{-1}\right]=(0)$. Thus (0) is a prime ideal in $T$.

Now $1^{\prime}$. and $2^{\prime}$. together allow us to perform a reasoning like that of lemma 6.1 in [4] with respect to the canonical injection $0 \rightarrow Q$ $\rightarrow Q_{2}$ and so we conclude $\hat{Q} \simeq Q_{2}$. Moreover $3^{\prime}$. tells us that $Q$ is an integral domain. We have only to prove that $Q$ is Noetherian. For this, let $\mathfrak{m}$ denote the maximal ideal of $Q$ and let $\mathfrak{b} \subset Q$ be a non-zero ideal; $\mathfrak{b} \hat{Q}$ can not be contained in the minimal prime of $\hat{Q}$ (since we have shown that it retracts to $(0)$ in $Q$ ), so it must be primary for $\mathfrak{m} \hat{Q}$ (since $\hat{Q}$ is 1 -dimensional). Choose an integer $k$ such that $\mathfrak{m}^{k} \hat{Q} \subset \mathfrak{b} \hat{Q}$. Then $\mathfrak{m}^{k} \subset \mathfrak{b}+\mathfrak{m}^{k+1}$ since $\mathfrak{m}^{k}$ is finitely generated we can use the Nakayama's lemma to conclude $\mathfrak{m}^{k} \subset \mathfrak{b}$, i.e. $\mathfrak{b}$ is $\mathfrak{m}$-primary. This means (0) and $\mathfrak{m}$ are the only prime ideals of $Q$, in $1^{\prime}$ we have seen that $\mathfrak{m}$ is finitely generated, therefore $Q$ is noetherian.

EXAMPLES 2.1. a) Let $\left(k, Q_{1}\right)$ be any flat couple with $k=\bar{k}$ a field and $Q_{1}$ a local artinian $k$-algebra which is not Gorenstein. If we apply the above construction to $\left(k, Q_{1}\right)$ we get a local (noetherian) 1-dimensional integral domain $Q$ whose formal fibers are not Gorenstein (in fact $Q_{(0)}$ is a field and $Q_{1}[[X]]_{\mathrm{m}_{1} Q_{1}[[X]]}$ is not Gorenstein since by hypothesis $Q_{1}$ is not so). All this in particular means that $Q$ is a local 1-dimensional C. M. domain having no Gorenstein modules of any rank (see [3] th. 5.2). (For another example of a ring with this feature see: D. Ferrand-M. Raynaud: Fibres formelles d'un anneau local noethérian, Ann. Sci. Ec. Norm. Sup. $4^{e}$ serie t. 3,1970$)$.

b) Let $\left(k, Q_{1}\right)$ be any flat couple such that $k=\bar{k}$ is a field and $Q_{1}$ is an artinian local $k$-algebra which is Gorenstein but not a complete intersection. If we apply the aforesaid construction to $\left(k, Q_{1}\right)$ we get a local (noetherian) 1-dimensional integral domain $Q$ which is Gorenstein and it is not expressible as a homomorphic image of a regular (local) ring. In fact $Q$ has completion $\hat{Q}=Q_{1}[[X]]$ which is Gorenstein (by [4] th. 9.8. since $Q_{1}$ is assumed to be Gorenstein by hypothesis), but it is 
not generically a complete intersection since $Q_{1}[[X]]_{\mathrm{m}_{1} Q_{1}[[X]]}$ is not a complete intersection (recall we are assuming $Q_{1}$ is not a complete intersection and apply Remark 2 after theorem 2 of [13]).

We can now conclude by applying Proposition 1.1.

c) Let $\left(k, Q_{1}\right)$ be any flat couple such that $k=\bar{k}$ is a field, $Q_{1}$ is an artinian local $k$-algebra which is Gorenstein and $d\left(Q_{1}\right)=2$ (this means $Q_{1}=k\left[\left[Z_{1}, \cdots, Z_{n}\right]\right] / \mathfrak{a}$ where $a$ is a $\left(Z_{1}, \cdots, Z_{n}\right)$-primary ideal generated by $n+2$ elements). If we apply the usual construction to $\left(k, Q_{1}\right)$ we get a local (noetherian) 1-dimensional integral domain $Q$ which is Gorenstein and can not be expressed as a homomorphic image of a regular (local) ring (that follows from $b$ ) since $d\left(Q_{1}\right)=2$ means in particular that $Q_{1}$ is not a complete intersection). We want to show that $Q$ does not have canonical algebra. We have $\hat{Q} \simeq k\left[\left[Z_{1}, \cdots, Z_{n}, X\right]\right] / a k\left[\left[Z_{1}, \cdots, Z_{n}, X\right]\right]$. For short we will write $k\left[\left[Z_{1}, \cdots, Z_{n}, X\right]\right]=S$ and $a k\left[\left[Z_{1}, \cdots, Z_{n}, X\right]\right]=\mathfrak{U}$.

Clearly $\mu(\mathfrak{U})=n+2$, let then $\left\{a_{1}, \cdots, a_{n+2}\right\}$ be a minimal system of generators for $\mathfrak{A}$. By [6] theorem 2.2.2 there exists the canonical algebra $A(\hat{Q})$ and precisely $A(\hat{Q}) \simeq H(E)$ where $E$ is the Koszul complex of $S$ with respect to $\left\{a_{1}, \cdots, a_{n+2}\right\}$. Let $\mathfrak{p}$ be the minimal prime of $\hat{Q}$, hence $\mathfrak{p} \cap Q=(0)$, and let $\mathfrak{B} \subset S$ be the prime ideal which is the image of $\mathfrak{p}$ in the canonical map Spec $\hat{Q} \rightarrow \operatorname{Spec} S$. We have $Q_{1}[[X]]_{\mathfrak{m}_{1} Q_{1}[[x]]} \simeq \hat{Q}_{p} \simeq$ $S_{\mathfrak{B}} / \mathfrak{A} S_{\mathfrak{\beta}}$. Moreover, the images $\tilde{a}_{i}$ of the $a_{i}$ 's in $S_{\mathfrak{B}}$, minimally generate $\mathfrak{X} S_{\mathfrak{P}}$. In fact, $\mu\left(\mathfrak{X} S_{\mathfrak{P}}\right) \leqslant \mu(\mathfrak{U})=n+2$ but, $\mu\left(\mathfrak{X} S_{\mathfrak{P}}\right)=n+1$ implies $Q_{1}[[X]]_{\mathfrak{m}_{1} Q_{1}[[X]]}$ is an almost complete intersection i.e. it is not Gorenstein and this is absurd (see [4] th. 9.8); on the other hand $\mu\left(\mathfrak{U} S_{\mathfrak{B}}\right)=n$ implies $Q_{1}[[X]]_{\mathfrak{m}_{1} Q_{1}[[X]]}$ is a complete intersection which is also absurd. Therefore, if we denote by $\tilde{E}$ the Koszul complex of $S_{\Re}$ with respect to $\left\{\tilde{a}_{1}, \cdots, \tilde{a}_{n+2}\right\}$ we have that $\tilde{E}=E_{\mathfrak{\wp}}$, so: $H(\tilde{E}) \simeq H\left(E_{\mathfrak{\beta}}\right) \simeq H(E)_{\mathfrak{\beta}} \simeq A(\hat{Q})_{\mathfrak{p}}$ (see [6] th. 2.4.7). Now since $S_{\mathfrak{B}}$ is a regular local ring and $\left\{\tilde{a}_{1}, \ldots, \tilde{a}_{n+2}\right\}$ is a minimal system of generators for $\mathfrak{A} S_{\mathfrak{B}}$, and since $\hat{Q}_{\mathfrak{p}} \simeq S_{\mathfrak{p}} / \mathfrak{Y} S_{\mathfrak{P}}$, we have $A(\hat{Q})_{\mathfrak{p}} \simeq A\left(\hat{Q}_{\mathfrak{p}}\right)$. All this will allow us to say that $Q$ has no canonical algebra. For this, suppose that $A(Q)$ exists, then by definition: $A(Q)^{\wedge} \simeq A(Q) \otimes_{Q} \hat{Q} \simeq A(\hat{Q})$. Moreover since $Q$ is an integral domain, $Q_{(0)}$ is a field and so $A(Q) \otimes_{Q}$ $Q_{(0)}$ is free (finite) over $Q_{(0)}$. From $A\left(\hat{Q}_{\mathfrak{p}}\right) \simeq A(\hat{Q})_{\mathfrak{p}}$ and $\mathfrak{p} \cap Q=(0)$ it follows

$$
\begin{aligned}
A\left(\hat{Q}_{\mathfrak{p}}\right) & \simeq A(\hat{Q})_{\mathfrak{p}} \simeq A(Q) \otimes_{Q} \hat{Q} \otimes_{\hat{Q}} \hat{Q}_{\mathfrak{p}} \simeq A(Q) \otimes_{Q} \hat{Q}_{\mathfrak{p}} \\
& \simeq A(Q) \otimes_{Q} Q_{(0)} \otimes_{Q_{(0)}} \hat{Q}_{\mathfrak{p}} \simeq \bigoplus_{\text {fin }} Q_{(0)} \otimes_{Q_{(0)}} \hat{Q}_{\mathfrak{p}} \simeq \bigoplus_{\text {fin }} \hat{Q}_{\mathfrak{p}} .
\end{aligned}
$$


Therefore $H_{1}(\tilde{E})$ is free over $\hat{Q}_{\mathfrak{p}}$.

Recall now that $\hat{Q}_{p}$ is a homomorphic image of the regular (local) ring $S_{\mathfrak{\beta}}$. That, together with $H_{1}(\tilde{E})$ free over $\hat{Q}_{\mathfrak{p}}$ implies, by [5] theorem 1.4.9, that $\hat{Q}_{\mathfrak{p}} \simeq Q_{1}[[X]]_{\mathrm{m}_{1} Q_{1}[[X]]}$ is a complete intersection, a contradiction. Therefore $A(Q)$ does not exist.

\section{BIBLIOGRAPHY}

[1] H. Bass: Injective dimension in noetherian rings. Trans. A.M.S. 102, 1962.

[2] J. Dieudonne: Topics in local algebra. Notre Dame Math. Lect. 10, 1967.

[3] R. Fossum, H. B. Foxby, P. Griffith and I. Reiten: Minimal injective resolution with application to dualizing modules and Gorenstein modules. Publ. Math. I.H.E.S. 45, 1975.

[4] S. Greco and P. Salmon: Topics in m-adic topologies. Erg. der Math. Springer Verlag 58, 1971.

[5] T. H. Gulliksen and G. Levin: Homology of local rings. Queen's Papers 20, 1969.

[ 6 ] J. Herzog: Komplexe, Aufösungen und Dualität in der lokalen Algebra. Habilitationsschrift (unpublished), Universität Regensburg, Regensburg, Germany.

[7] J. Herzog and E. Kunz: Der kanonische Modul eines C.M. Rings. Lect. Notes in Math. 238, 1971.

[ 8 ] E. Kunz: Almost complete intersections are not Gorenstein rings. J. of Algebra 28, 1974.

[ 9 ] L. Larfeldt and C. Lech: On flat couples (to appear).

[10] I. Reiten: The converse to a theorem of Sharp on Gorenstein modules. Proc. A.M.S. 32, 1972.

[11] G. Scheja: Über die Bettizahlen lokaler Ringe. Math. Ann. 155, 1964.

[12] R. Y. Sharp: Gorenstein modules. Math. Z. 115, 1970.

[13] K. Watanabe, T. Ishikawa, S. Tachibana and K. Otsuka: On tensor product of Gorenstein rings. J. Math. Kyoto Univ. 9, 1969. 